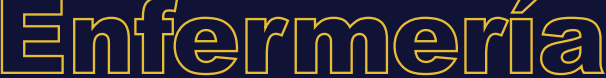 \\ Universitaria
}

\section{Adaptación del modo de autoconcepto en usuarios con diabetes tipo 2 de una unidad de primer nivel}

\section{Adaptation of the self-concept mode in users with diabetes type 2 in a first level unit}

\section{Adaptação do modo de autoconceito em usuários com diabetes tipo 2 de uma unidade de primeiro nível}

\author{
I.A. Medina-Fernández ${ }^{\mathrm{a} *}$, R. Gallegos-Torres ${ }^{\mathrm{b}}$, J. Candila-Celis ${ }^{\mathrm{c}}$ \\ a Facultad de Enfermería, Universidad Autónoma de Coahuila. Saltillo, Coahuila, México

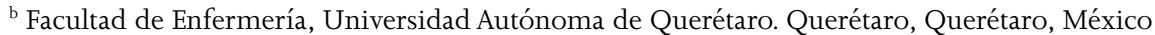 \\ ${ }^{c}$ Facultad de Enfermería, Universidad Autónoma de Yucatán. Mérida, Yucatán, México
}

Recibido: 6 noviembre 2017

Aceptado: 3 mayo 2018

\section{Resumen}

Objetivo: Comparar el nivel de adaptación del autoconcepto (NAA) en las personas que viven con Diabetes mellitus tipo 2 (DT2), en dos escenarios de una unidad de primer nivel.

Metodología: Investigación descriptiva, correlacional, comparativa y transversal con muestreo por conveniencia; se conformó por adultos con DT2 que asisten a control mensual y al grupo de ayuda mutua (GAM) en una unidad de primer nivel. Se aplicó cédula de datos generales e instrumento Viveros03 $(\alpha=.85)$; se realizó valoración antropométrica y glucemia capilar. El análisis de datos fue con SPSS v. 20. La comprobación de hipótesis fue mediante Shapiro-Wilk, T-student y Ji cuadrada.

Resultados: Se trabajó con 50 sujetos, 25 del GAM (Grupo A) y 25 de control ambulatorio (Grupo B). El NAA del grupo A fue $60 \%$ integrado y del B fue de $68 \%$ compensatorio, con diferencia significativa entre ambos grupos $(\mathrm{p}=0.02)$. Los estímulos contextuales como control glucémico, años de diagnóstico y tratamiento no demostraron dependencia $(\mathrm{p}>.05)$, contrario a los antecedentes familiares con diabetes y pertenecer al GAM en donde sí hubo relación $(\mathrm{p}=<.05)$.

Conclusión: El NAA fue mejor en el GAM, la valoración positiva del autoconcepto tuvo un mayor impacto, así mismo en el GAM, los antecedentes familiares son estímulos contextuales que permiten la adaptación de manera positiva.

Palabras clave: Teoría de enfermería; adaptación; diabetes mellitus tipo 2; México. 


\section{Abstract}

Objective: To compare the level of Adaptation of the Self-Concept (ASC) in persons suffering from Diabetes mellitus type 2 (DT2) in two locations at a first level unit. Methodology: This is a descriptive, correlational, comparative, and transversal research with a sample by convenience of adults with DT2 attending to a monthly control, and to a Mutual Support Group MSG, both in a first level unit. The general data register and the Viveros03 instrument $(\alpha=.85)$ were used. Anthropometric and capillary glycemic level assessments were performed. Data were analyzed through SPSS v.20. The hypothesis was verified using Shapiro-Wilk, T-student and Chi square tests.

Results: There were 50 participants, the 25 in the MSG were assigned to group A, while the other 25 constituted the ambulatory and group B. ASC in group A was $60 \%$ while $68 \%$ in group B; the difference was significant at $\mathrm{p}=0.002$. Contextual stimuli such as capillary glycemic level, and years of diagnosis and treatment did not show dependence $(\mathrm{p}>.05)$ contrary to family diabetes background, and belonging to the MSG, which did show an association ( $\mathrm{p}=.05)$.

Conclusion: ASC was better among those in the MSG, while family background promoted a better adaptation as well.

Keywords: Nursing theory; adaptation; diabetes mellitus type 2; Mexico.

\section{Resumo}

Objetivo: Comparar o nível de adaptação do autoconceito (NAA) nas pessoas que vivem com Diabetes mellitus tipo 2 (DT2), em dois cenários de uma unidade de primeiro nível. Metodologia: Pesquisa descritiva, correlacional, comparativa e transversal com amostragem por conveniência; conformou-se por adultos com DT2 que assistem a controle mensal e ao grupo de ajuda mutua (GAM) em uma unidade de primeiro nível. Aplicou-se cédula de dados generais e instrumento Viveros03 $(\alpha=.85)$; realizou-se valorização antropométrica e glicemia capilar. A análise de dados foi com SPSS v. 20. A comprobação de hipótese foi mediante Shapiro-Wilk, T-student e Ji cuadrada. Resultados: Trabalhou-se com 50 indivíduos, 25 do GAM (Grupo A) e 25 de controle ambulatório (Grupo B). O NAA do grupo A foi $60 \%$ integrado e do B foi de $68 \%$ compensatório, com diferença significativa entre ambos os grupos $(p=0.02)$. Os estímulos contextuais como controle glicêmico, anos de diagnóstico e tratamento não demonstraram dependência ( $\mathrm{p}>.05)$, contrário aos antecedentes familiares com diabetes e pertencer ao GAM onde houve uma relação $(\mathrm{p}=<.05)$.

Conclusão: O NAA foi melhor no GAM, a avaliação positiva do autoconceito teve um maior impacto, assim mesmo no GAM, os antecedentes familiares são estímulos contextuais que permitem a adaptação de maneira positiva.

Palavras chave: Teoria de enfermagem; adaptação; diabetes mellitus tipo 2; México.

\section{Introducción}

Actualmente, la salud ha considerado fenómenos que abarcan no sólo el aspecto biológico en el proceso patológico, sino que incluye también los aspectos de tipo psicológico y social ${ }^{1}$. Una de las patologías que tiene, además de la fuerte carga biológica, un importante vínculo personal y social, es la diabetes mellitus.

Año con año, la diabetes muestra un aumento en el número de casos, con un gran impacto en las poblaciones vulnerables debido a que produce cambios biológicos, psicológicos y sociales que han provocado una epidemia creciente en los países de ingresos medios y bajos ${ }^{2,3}$.

La Organización Mundial de la Salud (OMS) refiere que, en el 2013, en el mundo, se presentaron 382 millones de personas con diabetes, de los cuales el 90\% de los casos fueron del tipo 2; existen 246 millones de personas con diabetes que se encuentran en la zona urbana, 136 millones en zona rural y más del $80 \%$ vive en países de ingresos medios y bajos ${ }^{2,4}$. México se encuentra como uno de los países con mayor prevalencia de diabetes; los reportes de la Encuesta Nacional de Salud y Nutrición (ENSANUT) 
en el 2016 identificaron una prevalencia del 9.4\% de adultos mexicanos con la patología ${ }^{5,6}$, observaron un ligero aumento con respecto a la ENSANUT 2012; otro aspecto a resaltar es que el diagnóstico fue mayor en mujeres que en hombres. En el sur del país, en cambio, la prevalencia es mayor en hombres que en mujeres. Como antecedente, en el 2012, en Yucatán el 9.2\% de la población tuvo diabetes, este valor es ligeramente mayor que la media nacional en mujeres con un $11 \%$, la prevalencia en hombres fue de $7.2 \%{ }^{7}$.

Con base en lo anterior, se reconoce que la investigación en materia de diabetes aún tiene mucho camino por delante. En este sentido, se hace necesario estudiar la adaptación de las personas a la enfermedad, debido a que es un proceso que incluye la integración fisiológica, psicológica y social; dicha integración puede favorecer la plenitud del sistema, es decir, de la persona ${ }^{8}$. La adaptación de las personas para responder a una enfermedad crónica se vuelve un proceso complejo, que implica un balance entre las demandas de la situación y la habilidad del individuo?.

Una de las teorías de enfermería que expone la adaptación del individuo a la enfermedad es el modelo de Callista Roy, el cual propone cuatro formas de comportamiento a los que denomina "modos adaptativos" y la constituyen el modo fisiológico, modo de interdependencia, modo de función del rol y modo de autoconcepto. Este último se centra en los aspectos psicológicos y espirituales del sistema humano, el cual presenta los siguientes componentes: 1) el yo físico, el cual se manifiesta por las sensaciones corporales y la propia imagen; 2) el yo personal, que incluye el yo consciente, el yo ideal, yo moral, yo ético y yo espiritual, cuya necesidad subyacente del individuo es la integridad fisica ${ }^{10}$.

Roy, al hablar sobre los niveles de adaptación, señala tres: el primer nivel se denomina integrador, el cual describe las estructuras y las funciones del proceso de la vida en su totalidad para resolver necesidades del ser humano; el segundo nivel es el de compensar, en el cual el subsistema cognitivo y el regulador han sido activados por un desafío a los procesos integrados; el tercer nivel de adaptación es el comprometido, este sucede cuando los procesos integrados y compensatorios son inadecuados ${ }^{11}$.

Asimismo, describe tres tipos de estímulos ambientales, el estímulo focal es con lo que se enfrenta el individuo de manera más inmediata, reclama la mayoría de su atención y de energía de adaptación; los estímulos contextuales contribuyen positiva o negativamente a la fuerza del estímulo focal, por último se encuentran los estímulos residuales, cuyos efectos no son fácilmente conocidos ${ }^{12}$. En este sentido, entra en juego la adaptación del individuo a su enfermedad en todas sus esferas, lo que implica que el personal de enfermería conozca y comprenda dicho proceso para poder realizar el cuidado ${ }^{13}$.

Por otra parte, es importante referir que al investigar el nivel de adaptación en el modo de autoconcepto, se intenta evaluar el nivel de respuesta adaptativa a la enfermedad en función del reconocimiento o aceptación de la enfermedad misma, debido a que el primer paso para asumir el autocuidado es la adherencia al tratamiento. Es por ello, que en las unidades de primer nivel de atención se brinda un tratamiento integral a las personas que viven con Diabetes mellitus tipo 2 (DT2); también se implementan programas de autocontrol donde se consideran los aspectos de enseñanza del proceso de la enfermedad, el autocuidado, la dinámica familiar, apoyo físico, psicológico y social.

Con base en lo señalado, se hace necesario indagar la adaptación de las personas a la DT2, en una unidad de primer nivel. Dicha unidad está ubicada en una zona suburbana de Mérida, Yucatán, en la comunidad de "San José Tecoh", la cual brinda servicio a las localidades que se consideran de marginación social, con bajos niveles educativos y con alta prevalencia de obesidad y diabetes; en esta se ofrecen servicios integrales a familias, que son consideradas como población vulnerable social y económicamente ${ }^{14}$.

Por lo anterior, es necesario reconocer nuevos aspectos que deben ser incorporados en las formas de intervenciones integrales, así como la creación de estrategias entre profesionales de la salud y pacientes, que permitan la adaptación y los cambios actitudinales del individuo en el manejo y control de la enfermedad ${ }^{15}$.

En la unidad de salud ya señalada, se cuenta con grupos de ayuda mutua, los cuales son diversos y entre estos se encuentran los grupos de personas que padecen diabetes. En este grupo se ayuda a sus 
integrantes a alcanzar metas, entre ellas, el control del tratamiento y el metabólico, así como favorecer las relaciones sociales, promoción de la salud en diversos ámbitos y prevenir complicaciones de la enfermedad.

A partir de lo planteado anteriormente, el objetivo de esta investigación fue identificar el nivel de adaptación de autoconcepto en personas que viven con DT2 que asisten al grupo de ayuda mutua y en aquellos usuarios que asisten sólo a control ambulatorio en la Unidad Universitaria de Inserción Social San José Tecoh, Mérida, Yucatán, de manera tal que se reconozcan diferencias y similitudes entre ambos grupos, que puedan coadyuvar en el cuidado que brinda enfermería.

\section{Metodología}

El estudio fue de tipo descriptivo, correlacional, comparativo y trasversal. La población de estudio estuvo constituida por personas con DT2, de dos escenarios de la Unidad Universitaria de Inserción Social (UUIS), la primera integrada por adultos que asisten al grupo de ayuda mutua (GAM) [grupo A] y la segunda los que asisten solo a control glucémico mensual en la UUIS [Grupo B]. Se utilizó un muestreo no probabilístico por conveniencia, se consideró a las personas que tuvieron las siguientes características: sujetos diagnosticados con DT2, que contaron con tratamiento médico para la enfermedad, que asistieran al departamento de enfermería para control mensual, además de aquellos que eran miembros del GAM en la UUIS, con una asistencia mínima de 6 meses y que firmaran el consentimiento informado.

Para este estudio se utilizó el instrumento denominado Viveros03, que consta de 57 ítems que permiten valorar el nivel de autoconcepto de personas con enfermedades crónicas, desde un enfoque del modelo de adaptación de Callista Roy, el cual mide todos los componentes de dicho modo de autoconcepto, clasifica los niveles de adaptación según los siguientes rangos de 57-133 puntos nivel comprometido, 134-209 nivel compensatorio, 210-285 nivel integrado; el instrumento ha sido validado en población mexicana y tiene un alfa de Cronbrach de $.85{ }^{16}$.

Para identificar las variables socio demográficas y clínicas, se elaboró una cédula de datos que incluyó tres apartados: el primero incluía edad, género, estado civil, nivel económico, ocupación, escolaridad; el segundo, datos antropométricos y, por último datos clínicos en el que se consideró medicamentos de consumo, glucosa capilar, antecedentes hereditarios, antecedentes personales patológicos, antecedentes no patológicos.

Para medir las características clínicas se consideraron los niveles de glucosa plasmática, se tomaron en cuenta los cortes establecidos por la Norma Oficial Mexicana para la prevención, tratamiento y control de la diabetes mellitus en la atención primaria (NOM-015-SSA2-2010), se estableció que el paciente se encuentra controlado con la glucosa capilar cuando se obtuvo un valor de 70 a $130 \mathrm{mg} / \mathrm{dl}$ y descontrolado cuando es mayor de $130 \mathrm{mg} / \mathrm{dl}^{17}$.

Para calcular los puntos de corte del IMC, se tomó en cuenta la NOM-008-SSA3-2010 para el tratamiento integral del sobrepeso y la obesidad para personas con estatura estándar y de baja estatura. De la misma manera, se consideraron los criterios de riesgo de la circunferencia de cintura referidos por la Federación Internacional de Diabetes, tanto en hombres como en mujeres ${ }^{18,19}$.

La recolección de datos se llevó a cabo del mes de agosto al mes de noviembre del 2016 en la UUIS, con previa aceptación del protocolo de investigación por el comité de ética e investigación de la Facultad de Enfermería de la Universidad Autónoma de Yucatán; una vez identificados los participantes que cumplían con los criterios de inclusión de cada escenario, se les informó el objetivo del estudio, así como en qué consistiría su participación; los que aceptaron participar firmaron la hoja del consentimiento informado con el aval de un testigo y se procedió a la aplicación de la cédula de datos, valoración antropométrica y clínica, por último la aplicación del instrumento Viveros03.

El procesamiento de la información de los datos cuantitativos se realizó con el programa Statistic Package for the Social Science (SPSS) versión 20. Se utilizó estadística descriptiva para el instrumento 
Viveros03 y cédula de datos, se aplicó la frecuencia, media y desviación estándar con el fin determinar la puntuación del nivel de autoconcepto, las características sociodemográficas, clínicas y antropométricas.

Para evaluar la normalidad de las variables se aplicó la prueba de Shapiro-Wilk en ambos grupos; a fin de corroborar la hipótesis de que la adaptación de autoconcepto del grupo A es significativamente distinta a la del grupo B, se utilizó la prueba t de student para muestras no relacionadas, dado que la prueba de normalidad brindó una p>.05. Del mismo modo, para demostrar la relación de los estímulos contextuales (tratamiento, control glucémico en la DT2, antecedentes heredofamiliares, asistir al GAM) con el nivel de adaptación se utilizó la prueba de ji cuadrada.

Los aspectos éticos de la investigación fueron congruentes con lo establecido en los códigos de Núremberg y la Norma Oficial mexicana NOM-012-SSA3-2012, que establece los criterios para la ejecución de proyectos de investigación en seres humanos. De igual manera se observó lo señalado en la Ley General de Salud título quinto, capitulo único, así como lo establecido en la declaración de Helsinki en los aspectos de la atención que tienen relación con la investigación.

\section{Resultados}

La muestra estuvo compuesta por 50 participantes, repartidos entre el grupo A y B; el primero lo conformaron 25 personas que asistían al grupo de ayuda mutua y el segundo, 25 personas que se encontraban en control ambulatorio. El promedio de edad fue de 59.8 años en ambos grupos $(\mathrm{DE}=11.64 ;$ rango $=36-82)$. En relación con el sexo, en su mayoría fueron mujeres con un $78 \%$ $(n=39)$ y $22 \%(n=11)$ hombres (Tabla 1$)$.

Al cuestionar sobre los años de haber sido diagnosticados con diabetes, la muestra señaló una $\overline{\mathrm{x}}=10.38$ años ( $\mathrm{DE}=7.67$ años; rango $=1-30$ años), así mismo al realizarse las mediciones antropométricas la media obtenida del IMC fue de $29.90 \mathrm{~kg} / \mathrm{m}^{2}$ (DE=6.03 kg/ $\mathrm{m}^{2}$; rango $18.14-47.12 \mathrm{~kg} / \mathrm{m}^{2}$ ), la circunferencia de cintura para hombres obtuvo una $\overline{\mathrm{x}}=108.09 \mathrm{~cm}$ y una $\mathrm{DE}=31.52$ y en mujeres la $\overline{\mathrm{x}}=97.64$ y una $\mathrm{DE}=9.772$, además la glucemia capilar tuvo una $\overline{\mathrm{x}}=150.98 \mathrm{mg} / \mathrm{dl}$ $(\mathrm{DE}=53.07 \mathrm{mg} / \mathrm{dl} ;$ rango=88-319 mg/dl) (Tabla 2).

En relación al IMC, el escenario A obtuvo 64\% (16) de obesidad y $24 \%$ (6) de sobrepeso, comparado con el escenario B, donde el $56 \%(14)$ tienen obesidad y $24 \%$ (6) sobrepeso. El riesgo cardio metabólico en el escenario A fue del $50 \%$ (1) en los hombres y el $87 \%(20)$ en las mujeres; en cambio en el escenario B el 77.8\%(7) de los hombres y el 100\% (16) de las mujeres presentaron el riesgo. Con base al control glucémico el 44\% (11) del escenario A y el 40\% (10) del B se encuentran controlados.

En relación con el tratamiento, los participantes indicaron, con un $84 \%(\mathrm{n}=21)$ en el grupo $\mathrm{A}$ y un $76 \%(19)$ en el grupo B que consumen hipoglucemiantes orales, ya sea sulfunilureas o biguaninas; en cuanto al consumo mixto de insulina-hipoglucemiante oral,

\begin{tabular}{|c|c|c|c|c|}
\hline \multirow[b]{2}{*}{ Variable } & \multicolumn{2}{|c|}{$\begin{array}{c}\text { Grupo A } \\
(n=25)\end{array}$} & \multicolumn{2}{|c|}{$\begin{array}{c}\text { Grupo B } \\
(n=25)\end{array}$} \\
\hline & $\mathbf{F}$ & $\%$ & $\mathbf{F}$ & $\%$ \\
\hline \multicolumn{5}{|l|}{ Sexo } \\
\hline Hombre & 2 & 8 & 9 & 36 \\
\hline Mujer & 23 & 92 & 16 & 64 \\
\hline \multicolumn{5}{|l|}{ Ocupación } \\
\hline Ama de casa & 22 & 88 & 14 & 56 \\
\hline Trabajo Independiente & 2 & 8 & 9 & 36 \\
\hline Obrero & 1 & 4 & 1 & 4 \\
\hline Pensionado & 0 & 0 & 1 & 4 \\
\hline \multicolumn{5}{|l|}{ Estado Civil } \\
\hline Soltero & 4 & 16 & 2 & 8 \\
\hline Casado & 16 & 64 & 14 & 56 \\
\hline Unión libre & 2 & 8 & 6 & 24 \\
\hline Divorciado & 2 & 8 & 3 & 12 \\
\hline Viudo & 1 & 4 & 0 & 0 \\
\hline \multicolumn{5}{|l|}{ Nivel de escolaridad } \\
\hline No escolarizado & 3 & 12 & 8 & 32 \\
\hline Primaria trunca & 9 & 36 & 2 & 8 \\
\hline Primaria completa & 5 & 20 & 4 & 16 \\
\hline Secundaria trunca & 6 & 24 & 7 & 28 \\
\hline Secundaria completa & 0 & 0 & 2 & 8 \\
\hline Preparatoria & 2 & 8 & 1 & 4 \\
\hline Licenciatura & 0 & 0 & 1 & 4 \\
\hline \multicolumn{5}{|l|}{ Religión } \\
\hline Ninguna & 2 & 8 & 1 & 4 \\
\hline Católica & 20 & 80 & 19 & 76 \\
\hline Testigo de Jehová & 1 & 4 & 3 & 12 \\
\hline Cristiana & 2 & 8 & 0 & 0 \\
\hline Presbiteriana & 0 & 0 & 2 & 8 \\
\hline
\end{tabular}


Tabla 2. Descripción de las variables antropométricas y clínicas en ambos grupos $(\mathbf{n}=\mathbf{5 0})$

\begin{tabular}{|c|c|c|c|c|c|}
\hline Variable & Grupo & $\overline{\mathbf{x}}$ & DE & V. Mínimo & V. Máximo \\
\hline \multirow[t]{2}{*}{ Peso (Kg) } & $\mathrm{A}$ & 66.79 & 13.44 & 46.00 & 93.00 \\
\hline & $\mathrm{B}$ & 70.99 & 20.54 & 44.00 & 133.00 \\
\hline \multirow[t]{2}{*}{ IMC } & A & 30.14 & 6.02 & 18.14 & 43.03 \\
\hline & B & 29.67 & 6.69 & 21.36 & 47.12 \\
\hline \multirow[t]{6}{*}{ Cintura $(\mathrm{cm})$} & $\mathrm{A}$ & 96.88 & 10.50 & 80.00 & 115.00 \\
\hline & Hombres & 92.00 & 5.65 & 88.00 & 96.00 \\
\hline & Mujeres & 97.30 & 10.80 & 80.00 & 115.00 \\
\hline & B & 103.00 & 21.99 & 84.00 & 180.00 \\
\hline & Hombres & 11.67 & 34.04 & 84.00 & 180.00 \\
\hline & Mujeres & 98.12 & 8.38 & 85.00 & 115.00 \\
\hline \multirow[t]{2}{*}{ Años de diagnóstico } & A & 11.96 & 7.70 & 1.00 & 30.00 \\
\hline & B & 8.80 & 7.46 & 1.00 & 25.00 \\
\hline \multirow[t]{2}{*}{ Glucemia capilar (mg/dl) } & A & 138.76 & 35.05 & 92.00 & 225.00 \\
\hline & B & 163.20 & 64.89 & 88.00 & 319.00 \\
\hline
\end{tabular}

éste fue referido por un $16 \%(n=4)$ en el grupo A y en el B un 24\% $(n=6)$. Con respecto a los antecedentes patológicos, la prevalencia de hipertensión arterial sistémica fue referida en un $68 \%(n=17)$ para el escenario A y $64 \%(n=16)$ en el escenario B.

En la Gráfica 1 se observan las diferencias de medias de las puntuaciones de acuerdo al instrumento Viveros03, que permite valorar el nivel de respuesta adaptativa a la DT2 (modo de autoconcepto según dimensiones) en la muestra estudiada y en la Gráfica 2 el nivel de adaptación por escenario.

Para la comprobación de la hipótesis "la adaptación de autoconcepto del grupo A es distinta de manera significativa a la del grupo B". Se aplicó la prueba t de student para una muestra a fin de explorar las diferencias de medias del nivel de adaptación de autoconcepto y sus componentes, se identificó que el NAA es diferente en los grupos, lo que lleva a aceptar la hipótesis del investigador ( $t=3.391, p<.001)$.

Por otra parte, para verificar la hipótesis que plantea los estímulos contextuales (tratamiento, control glucémico en la DT2, antecedentes heredofamiliares, periodo de tiempo de vivir con DT2, asistir al GAM), se asocian con el nivel de adaptación de autoconcepto, se utilizó la prueba Ji cuadrado con corrección (cuando al menos el valor de una frecuencia esperada es menor que 5); los datos obtenidos indicaron que hubo asociación con antecedentes heredofamiliares $\left(x^{2}=7.42, \mathrm{p}=.024\right)$ y con asistir al GAM $\left(\mathrm{x}^{2}=9.815, \mathrm{p}=.007\right)$; no hubo asociación con las variables de control glucémico $\left(x^{2}=2.31, p=.315\right)$, periodo de tiempo de vivir con DT2 $\left(x^{2}=6.50\right.$, $\mathrm{p}=.771)$ y tratamiento $\left(\mathrm{x}^{2}=.833, \mathrm{p}=.659\right)$.

Gráfica I. Comparación de medias de las puntuaciones del Yo personal y yo físico por grupo* $n=50$

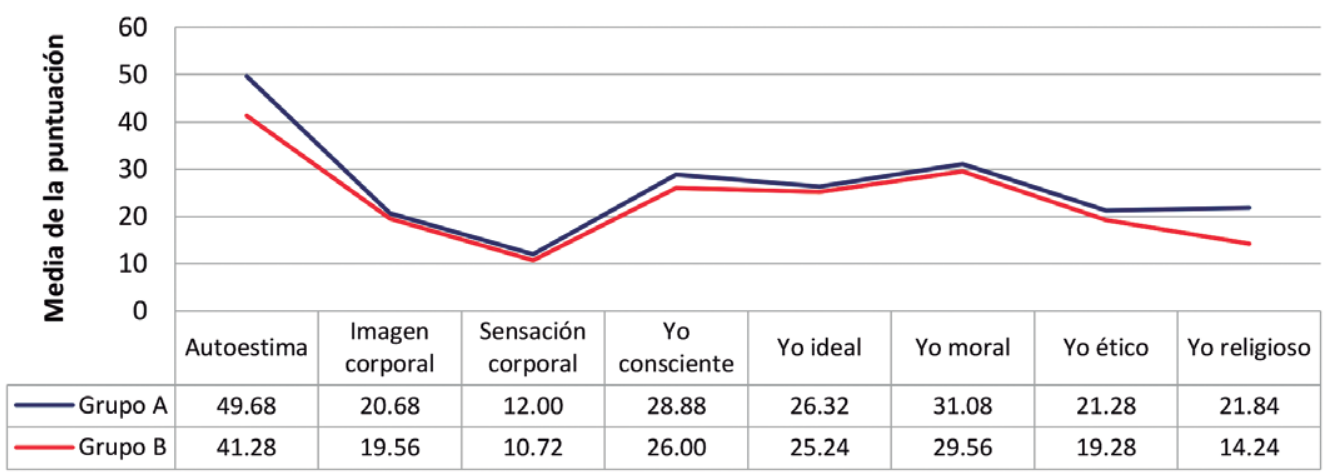

${ }^{*}$ Conforme al instrumento Viveros $03^{16}$ 


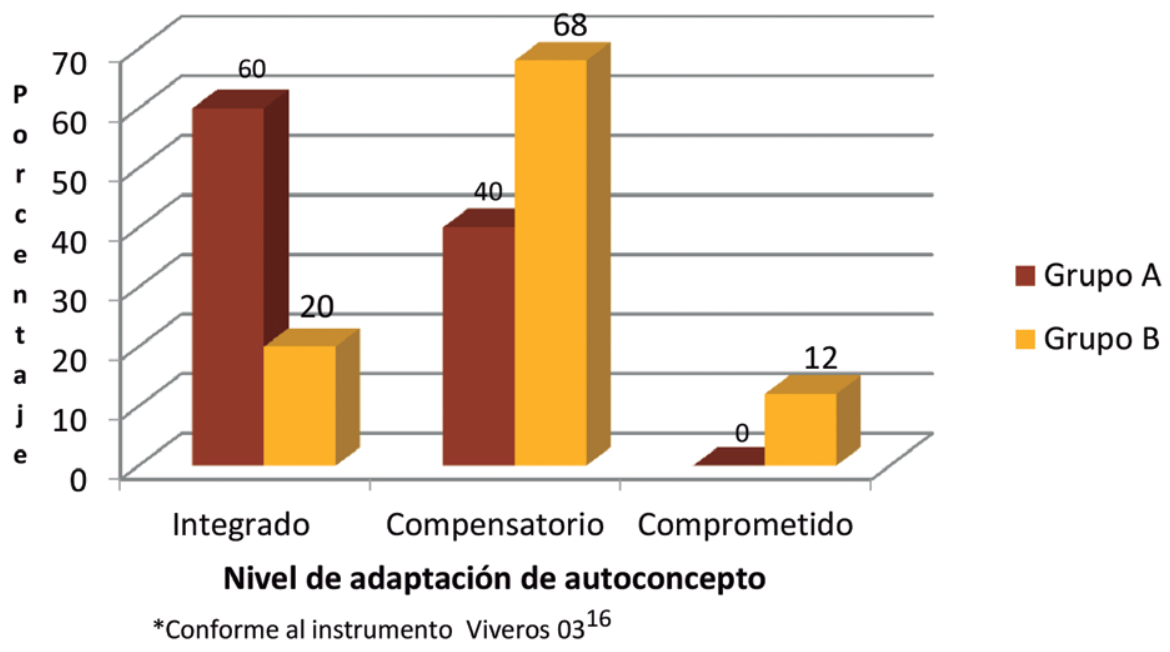

\section{Discusión}

Los resultados de las características clínicas y antropométricas de los participantes mostraron, con respecto a los años de diagnóstico, una media de 10 años; hay presencia de sobrepeso y obesidad, por lo tanto, el riesgo cardiovascular es alto en ambos grupos; de igual manera la media de la glucemia capilar se encontró en el parámetro descontrolado. Las características antropométricas antes mencionadas fueron similares a estudios realizados en San Luis Potosí (México), Brasil y Japón, de estos la media de la glucosa fue mayor en el estudio realizado en México ${ }^{20-22}$.

El nivel de adaptación de autoconcepto fue mejor en el grupo de ayuda mutua con $60 \%$; el $50 \%$ de los que tienen un buen control glucémico obtuvieron mejores puntuaciones. Esta situación concuerda con el estudio realizado en México a las personas con diabetes que asisten al grupo de ayuda mutua, cuyo resultado se ubicó en un 75\% con un autoconcepto alto ${ }^{22}$. Por otra parte, en el 55.3\% de las personas que asisten a una clínica de atención integral en Nicaragua, se obtuvo un nivel de adaptación integra$\mathrm{do}^{23}$. Sin embargo, los resultados del ensayo clínico realizado en un Hospital de Irán, el grupo de casos obtuvo un $49.2 \%$ de autoconcepto alto, comparado con el grupo control en donde el 11.3\% se encontró en esta categoría ${ }^{24}$. Cabe resaltar que no fueron considerados aspectos religiosos y culturales en dichas investigaciones. En este sentido la red de apoyo entre los participantes crea un ambiente social saludable, en donde se plasman metas y responsabilidades que hacen que se cumpla la necesidad humana de identidad de grupo.

En la presente investigación se evidenció estadísticamente que el nivel de adaptación a la enfermedad está relacionada con la participación en el grupo de apoyo $(\mathrm{t}=3.391, \mathrm{p}<0.05)$. No es posible comparar este dato puesto que no se han realizado estudios en donde se determine la importancia del autoconcepto en los grupos de ayuda mutua, pero sí se ha identificado que la educación de autoconcepto en las personas no fue significativa $(\mathrm{p}=<0.05)^{24}$. Un estudio plantea que existen diferencias significativas en el autoconcepto social en pacientes con diabetes de acuerdo a su adherencia al tratamiento $(\mathrm{p}=<0.05)^{25}$. Dichos resultados se explican por Vilaníi ${ }^{26}$, quien retoma a Tretini, este sostiene que el grupo se vuelve importante por el apoyo a sus miembros, contribuye a fortalecer sus metas, las cuales son resultantes del proceso interactivo del grupo, pero no debe ser abordado desde un enfoque educativo sino desde las habilidades para la vida con enfoque integral.

Dentro de este marco, la adaptación de autoconcepto no demostró dependencia con el control glucémico ( $\mathrm{p}=0.315)$. Por otro lado, en los estudios realizados en Irán, Japón y Brasil no hallaron correlación con esta variable $(\mathrm{p}=0.08),(\mathrm{p}=0.607)$ y $(\mathrm{p}=0.73)$, respectivamente ${ }^{27,20,21,}$, con esto se puede señalar que la adaptación de autoconcepto no influye en el control glucémico, aunque se observó que algunas 
de las personas que tienen niveles de glucemia fuera del parámetro normal presentaron un nivel de autoconcepto integrador, lo que es probablemente un mecanismo de afrontamiento a la enfermedad.

Con respecto a los años de diagnóstico y adaptación de autoconcepto no hubo relación entre las variables. Dichos resultados fueron similares a los reportados en el estudio realizado por Lazcano y Salazar $^{28}$, en un hospital público de Monterrey, México, en el cual se indica que los años diagnóstico no influyeron en la adaptación psicosocial $(\mathrm{p}=0.071)$; que coincide también con el estudio realizado por Portilla $^{25}$ en Perú ( $\mathrm{p}=0.089$ ). Lo anterior se relaciona con el hecho de que la persona con diabetes sufre a menudo cambios emocionales y tienen conceptos negativos de sí mismos, que influyen en las estrategias que permiten mantener la adaptación.

Otro hallazgo fue que la adaptación de autoconcepto tiene dependencia con tener algún antecedente familiar de la enfermedad $(\mathrm{p}=0.024)$. Este resultado es similar al estudio realizado en Irán $(\mathrm{p}=0.08)^{23}$, en donde se muestra que las experiencias previas facilitan la adaptación mediante el apoyo familiar para poder sobre llevar la enfermedad.

Las confirmaciones antes mencionadas forman parte de las respuestas adaptativas psicosociales, las cuales son útiles para el manejo de la patología, pues integran la experiencia de la enfermedad crónica con el concepto de sí mismo y la identidad personal, así como la posibilidad de integrar el cambio personal y los cambios del estilo de vida en las relaciones personales ${ }^{13 ;}$ por este motivo el grupo de apoyo es importante porque las personas encuentran puntos en común con sus similares, que les permite crear un ambiente de entendimiento, compañerismo y apoyo social, esto facilita la adaptación a la enfermedad.

Con base en lo anterior, el quehacer de enfermería debe romper paradigmas que generen nuevos cuidados de intervención, no sólo en el modo de adaptación fisiológico, sino también en el subsistema cognitivo (habilidades humanas de pensar, sentir y actuar a través del procesamiento de la información), con el fin de influir positivamente en la salud mental y física de las personas ${ }^{29}$.

\section{Conclusiones}

El presente estudio contribuye a la aplicación de la teoría de enfermería de rango medio del denominado modelo de adaptación de Callista Roy, que permite la aplicabilidad en personas que padecen diabetes, cuyos resultados, hacen que el ejercicio de enfermería tenga fundamentos científicamente comprobados, siendo útil para la práctica comunitaria, por ejemplo: en grupos como los de ayuda mutua, se puede favorecer el cumplimiento de las metas establecidas.

Se determinó que, las personas con DT 2 que asisten al grupo de ayuda mutua en la UUIS San José Tecoh, presentan un mejor nivel de adaptación de autoconcepto, es decir las intervenciones de enfermería son integrales porque tienen un impacto en el aspecto psicológico de la persona, les permite el verse a sí mismos de manera positiva, obtienen medias mayores en todos los componentes del modo de autoconcepto, con respecto a los pacientes que solo asisten a control ambulatorio.

Se comprobó que el grupo de ayuda mutua y tener un familiar con diabetes es un estímulo contextual que permite la adaptación de autoconcepto de manera positiva. Así mismo es posible establecer que el grupo de ayuda mutua fortalece las metas mediante el proceso de interacción con las personas que tienen DT 2, además con el equipo multidisciplinario que brinda la intervención, da como resultado la creación de experiencias positivas, la mejora de la salud mental y la obtención de comportamientos saludables.

\section{Responsabilidades Éticas}

Protección de personas y animales. Los autores declaran que en esta investigación no se han realizado experimentos en seres humanos ni en animales.

Confidencialidad. Los autores declaran que este trabajo no contiene ningún dato que permita identificar a los participantes.

Derecho a la privacidad y consentimiento informado: Los autores han obtenido el consentimiento informado de los participantes. 
Financiamiento. Ninguno

Conflicto de intereses. Ninguno

\section{Referencias}

1. Arrivillaga-Quintero M, Correa-Sánchez D, Salazar-Torres IC. Psicología de la salud. Abordaje integral de la enfermedad crónica. 2a ed. Bogotá: Manual Moderno; 2007. P.53.

2. Federación Internacional de Diabetes. Atlas de Diabetes. Bélgica: FID; 2013.[consultado 3 marzo 2016] Disponible en: https://bit.ly/2sdfAwf

3. International Diabetes Federation. Diabetes: A global Health and Development Challenge [Internet]. Brussels: International Diabetes Federation. [Consultado 3 marzo 2016]. Disponible en: https://bit.ly/2MYBYXC

4. Organización Mundial de la Salud. Diabetes. Datos y Cifras. Nota descriptiva N. ${ }^{\circ} 312$. Ginebra: OMS; 2012 [consultado 1 septiembre 2015]. Disponible en: https://bit.ly/1pNe2SA

5. Hernández-Avila M, Rivera-Dommarco J, Shamah-Levy T et al. Encuesta Nacional de Salud y Nutrición de Medio Camino 2016. Informe final de resultados. Cuernavaca: Instituto Nacional de Salud Pública. 2016.

6. Hernández- Ávila M, Gutiérrez JP. Evidencia para la política pública en salud. Diabetes mellitus: la urgencia de reforzar la respuesta en políticas públicas para su prevención y control. Cuernavaca: Instituto Nacional de Salud Pública; 2012.

7. Romero M, Shamah-Levy T, Franco A, et al. Encuesta Nacional de Salud y Nutrición 2012. Resultados por entidad federativa. Yucatán. Cuernavaca: Instituto Nacional de Salud Pública; 2013. P.44-65.

8. Villamizar-Carvajal B, Durán- de Villalobos MM. Modelo de adaptación de Roy en un ensayo clínico controlado. Av. enferm. 2012; 30(2):97-107.

9. García-Ajanel AV. Adaptación familiar y social del paciente diabético (estudio realizado en el patronato del diabético de la cabecera departamental de Quetzaltenango). [Tesis]. Quetzaltenango: Universidad Rafael Landívar; 2012.

10. Roy C. El modelo de adaptación de Roy en el contexto de los modelos de enfermería, con ejemplos de aplicaciones y dificultades. Cult. cuid. 2000; IV (7/8):139-59. https: / /doi.org/10.14198/cuid.2000.7-8.17

11. Ramírez-Ochoa MC, García-Campos ML, Alarcón-Rosales MA. Nivel de adaptación: rol e interdependencia de pacientes en diálisis peritoneal continua ambulatoria. Rev enferm Inst Mex Seguro Soc. 2008;16(3):145-53.

12. Phillips KD. Modelo de adaptación. En: Raile-Alligood M, Marriner-Tomey A. Modelos y teorías en enfermería. $7^{\text {a }}$ ed. Barcelona, España: Elseiver; 2011. p. 335-65.

13. Riffo-Luengo S, Salazar-Molina A. Aplicación del proceso de enfermería según el Modelo de Roy en pacientes con Diabetes Mellitus. Enferm glob. 2008; 7 (3): 13 pantallas.

14. Dájer Abimerhi AFJ. Programa institucional prioritario: Articulación y consolidación de las escuelas preparatorias, facultades, campus, unidades multidisciplinarias, unidad académica con interacción comunitaria y del centro de investigación. En: Informe de la gestión 2011, el camino de la transformación institucional a 2020. Mérida: UADY; 2011 : pp. 85-7.

15. Moore S, Shiell A, Hawe P, et al. The privileging of communitarian ideas: Citation practices and the translation of social capital into public health research. Am J Public Health. 2005; 95 (8): 1330-7. https://doi.org/10.2105/AJPH.2004.046094

16. Viveros-Salgado M, Díaz-Guerrero R. Instrumento Viveros 03 para Medir el Autoconcepto de Personas con Enfermedades Crónicas. Desarrollo cientif enferm. 2004; 12 (4): 113-7.

17. Secretaria de Salud. Norma Oficial Mexicana NOM-015-SSA2-2010, Para la prevención, tratamiento y control de la diabetes mellitus. Ciudad de México: DOF; 2010. 
18. Secretaría de Salud. Norma Oficial Mexicana NOM-008-SSA3-2010, Para el tratamiento integral del sobrepeso y la obesidad. Ciudad de México: DOF; 2010.

19. Zimmet P, Alberti G, Shaw J. Nueva definición mundial de la FID del síndrome metabólico: argumentos y resultados. Diabetes Voice. 2005; 50(3):31-3.

20. Nozaki T, Morita C, Matsubayashi S, et al. Relation between psychosocial variables and the glycemic control of patients with type 2 diabetes: A cross-sectional and prospective study. Biopsychosoc Med. 2009;3(4):1-8. https://doi.org/10.1186/1751-0759-3-4

21. Silva-Fuscaldi FS, Sartore-Balsanelli ACS, Alves-Grossi SAA. Lócus de controle em saúde e autoestima em portadores de diabetes mellitus tipo 2. Rev. Esc. Enferm. USP. 2011 ; 45 (4): 855-61. http://dx.doi.org/10.1590/S0080-62342011000400009

22. Díaz-Oviedo A, Betancourt-Esparza C, Cheverría-Rivera S, et al. Diabetes Mellitus: un estudio sobre el nivel de adaptación fisiológica y de autoconcepto en pacientes que participan en un grupo de apoyo. PAG rev. iberoam. producción académica gest. educ.. 2014; 1 (1): 28 páginas.

23. Correa-Morales JD. Autoconcepto de la diabetes mellitus en pacientes que asisten a la clínica de atención integral en el centro de salud Jorge Sinforoso Bravo. Municipio de Granada, Silais Granada. Periodo octubre-diciembre 2008 [Tesis]. Managua: Universidad Nacional Autónoma de Nicaragua; 2010.

24. Samadi N, Safavi M, Mahmoodi M. Impact of Quality of Life Education on Self-Concept among Type 2 Diabetes Patients. J Diabetes Metab. 2011; 2 (5): 5 pages. https://doi.org/10.4172/2155-6156.1000132

25. Portilla-García LA. Calidad de vida y autoconcepto en pacientes con diabetes con y sin adherencia al tratamiento. [Tesis]. Lima: Universidad Mayor de San Marcos; 2011.

26. Vilaní M, Leite T, Venícios M. Grupo como estrategia de cuidar a la luz del modelo de Adaptación de Roy. Enferm Integral. 2006; (76): 33-7.

27. Pourhaji F, Delshad MH, Ammari AA, et al. Foot-Care Self Efficacy Beliefs, Physical Self-Concept and actual Foot- Care Behavior in People with Diabetes Mellitus. Int J Musculoskelet Pain Prev 2016; 1(3): 101-7.

28. Lazcano-Ortíz M, Salazar-González BC. Adaptación en pacientes con diabetes Mellitus Tipo 2, según Modelo de Roy. Aquichan. 2009; 9(3): 236-45.

29. Gutiérrez-López C. Escala de medición del proceso de afrontamiento y adaptación de Callista Roy: una propuesta metodológica para su interpretación. Hallazgos- Revista de Investigaciones. 2009; 6 (12): 201-13. 